\title{
Pulsed Nuclear Pumping and Spin Diffusion in a Single Charged Quantum Dot
}

\author{
Thaddeus D. Ladd, ${ }^{1,2, \text { | }}$ David Press,${ }^{1}$ Kristiaan De Greve, ${ }^{1}$ Peter L. McMahon, ${ }^{1}$ Benedikt Friess,,${ }^{1,3}$ \\ Christian Schneider, ${ }^{3}$ Martin Kamp, ${ }^{3}$ Sven Höfling, ${ }^{3}$ Alfred Forchel, ${ }^{3}$ and Yoshihisa Yamamoto ${ }^{1,2}$ \\ ${ }^{1}$ E. L. Ginzton Laboratory, Stanford University, Stanford, California 94305, USA \\ ${ }^{2}$ National Institute of Informatics, Hitotsubashi 2-1-2, Chiyoda-ku, Tokyo 101-8403, Japan \\ ${ }^{3}$ Technische Physik, Physikalisches Institut, Wilhelm Conrad Röntgen Research Center for \\ Complex Material Systems, Universität Würzburg, Am Hubland, D-97074 Würzburg, Germany
}

\begin{abstract}
We report the observation of a feedback process between the nuclear spins in a single charged quantum dot and its trion transition, driven by a periodic sequence of optical pulses. The pulse sequence intersperses off-resonant ultrafast pulses for coherent electron-spin rotation and resonant narrow-band optical pumping. The feedback manifests as a hysteretic triangle-like pattern in the free-induction-decay of the single spin. We present a simple, quasi-analytic numerical model to describe this observation, indicating that the feedback process results from the countering effects of optical nuclear pumping and nuclear spin-diffusion inside the quantum dot. This effect allows dynamic tuning of the electron Larmor frequency to a value determined by the pulse timing, potentially allowing more complex coherent control operations.
\end{abstract}

Optically controlled quantum dots (QDs) are in many ways similar to atomic systems, and are therefore often regarded as strong candidates for solid-state quantum information processing. However, one key feature distinguishing QDS in group III-V semiconductors from atomic systems is the presence of a large nuclear-spin ensemble [1]. Nuclear spins cause adverse effects such as inhomogeneous broadening and non-Markovian decoherence processes. However, nuclear spins may play useful roles as well. Although methods to use QD nuclear spins directly as a quantum memory remain challenging due to the difficulty of achieving sufficiently high levels of nuclear polarization, nuclear spins may provide novel methods for the dynamic tuning and locking of electron spin resonances for electrons trapped in QDs.

Several examples of manipulating nuclei to improve electron spin coherence have recently been observed. In electrically controlled double QDs, transition processes between electron singlet and triplet states allow the manipulation of interdot nuclear spin polarizations, improving coherent control [2, 3, 4]. In single QDs under microwave control, nuclear effects dynamically tune the electron spin resonance to the applied microwave frequency [5. Tuning effects are also observed in two-color continuous-wave (CW) laser experiments, in which the appearance of coherent electronic effects such as population trapping are modified by nonlinear feedback processes with nuclear spins 6, 7]. Finally, nuclear spins have been shown to dynamically bring ensembles of inhomogeneous QDs into spin-resonance with a train of ultrafast pulses [8, 9].

Here, we describe a related but different manifestation of the non-Markovian dynamics occurring between a single electron in a QD and the nuclear bath with which it interacts, with new possibilities for use in controlling nuclear effects. The effect occurs when measuring the familiar "free-induction decay" (FID) of a single spin in a single QD under pulsed control. The Larmor frequency of the electron spin is dynamically altered by the hyperfine interaction with QD nuclei; the nuclear polarization is in turn altered by the measurement results of the FID experiment. The result is a feedback loop in which the nuclear hyperfine field stabilizes to a value determined by the timing of the pulse sequence. In what follows, we show the experimental manifestation of this feedback loop and present a numerical model for the effect.

The FID or Ramsey interferometer experiment proceeds by tipping a single electron spin from the pole of its Bloch sphere onto the equator with a $\pi / 2$ rotation, allowing it to precess for a time $\tau$, and then tipping it with a second $\pi / 2$ rotation into a state with polarization depending sinusoidally on $\tau$. We accomplish this experiment in a single, charged, self-assembled InAs QD using periodically applied pairs of ultrafast optical pulses, interspersed with optical pumping. The molecular-beamepitaxially grown QDs are centered in a planar microcavity with 24 and 5 pairs of GaAs/AlAs $\lambda / 4$ mirror pairs below and above the cavity, respectively. The cavity quality factor was roughly $Q \approx 200$, and the single-sided design of the cavity directed most emission towards the collection optics. A layer of Si dopants placed $10 \mathrm{~nm}$ below the QDS served to probablistically dope the QDS with electrons. Several prior experiments have shown that single ultrafast pulses achieve coherent, single-spin rotations in charged QDs [10, 11, 12. The rotation occurs on picosecond timescales, which is effectively instantaneous in comparison to electron-spin decoherence times or nuclear spin dynamics. The optical pumping in our experiment, previously described in Ref. 11, is accomplished via a trion transition. It serves to repeatedly re-initialize the spin into one particular polarization state prior to the ultrafast pulses. Spin measurement is accomplished by single-photon-counting during the optical pumping step; the photon count-rate is proportional to the probability of exciting a trion state. This count-rate averaged over many sequential experiments is expected to vary sinu- 
(a) Scanning Forward

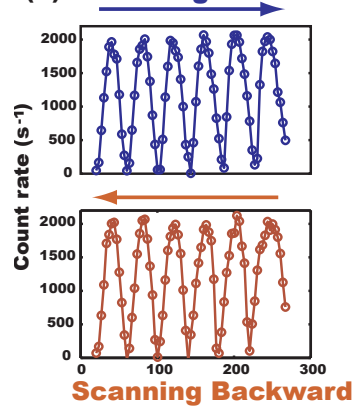

(b)

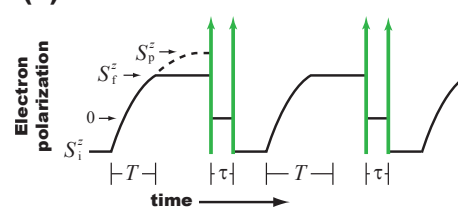

(c)

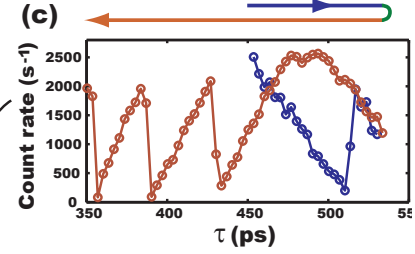

FIG. 1: (Color online) (a) Experimental Ramsey fringe countrate as a function of two-pulse time delay $\tau$, measured using the techniques of Ref. 11] This data was taken at a magnetic field of $4 \mathrm{~T}$, but similar effects are seen at different magnetic fields. (b) Average electron polarization as a result of the periodic pulse sequence used to generate this data. Optical pumping increases the polarization for a duration $T=26 \mathrm{~ns}$. The saturation polarization, which would be reached for $T \rightarrow \infty$ (dashed line) is $S_{\mathrm{p}}^{z}$; in time $T$ only polarization $S_{\mathrm{f}}^{z}$ is reached. After pumping and a short delay, a picosecond pulse indicated by a green (grey) arrow nearly instantaneously rotates the electron spin to the equator of the Bloch sphere $\left(\left\langle S^{z}\right\rangle=0\right)$; a time $\tau$ later a second pulse rotates the spin to achieve electron polarization $S_{\mathrm{i}}^{z}$, depending on the amount of Larmor precession between the pulses. The theoretical count-rate $C(\omega, \tau)$ of Eq. (1) is found as $S_{\mathrm{f}}^{z}-S_{\mathrm{i}}^{z}$ in steady-state conditions. (c) Experimental Ramsey fringe count-rate as $\tau$ is continuously scanned forward and then backward, showing clear hysteresis.

soidally with $\tau$, resulting in "Ramsey fringes," and to decay on a timescale called $T_{2}^{*}$ due to dephasing or decoherence effects.

In contrast to earlier work [1], the resonant laser used to optically initialize the single spin is gated off during the free precession of the electron spin using an electrooptic modulator. This eliminates the previously observed source of decay of Ramsey fringes, leading to an expected longer $T_{2}^{*}$ decay. In particular, a Markovian picture of nuclear spin noise processes predicts a Gaussian decay with $T_{2}^{*}$ timescale on the order of a few nanoseconds.

However, such a Gaussian decay was not observed. Figure 11 shows the result of the FID experiment. The top three traces show the fringes seen as $\tau$ is scanned in the forward direction (increasing the delay $\tau$ by moving a retroreflector outwards with a scanning stage), and the bottom three correspond to scanning in the backward direction (reducing the delay $\tau$ by moving the retroreflector inwards). The oscillatory fringes, rather than decaying, evolve into a sawtooth pattern at high values of $\tau$ [18, and show hysteresis depending on the direction in which

$\tau$ is changed. The break between traces in Fig. 1(a) is due to the need to manually move the scanning stage.

This data is the result of two competing processes: changes in the average nuclear hyperfine shift $\omega$ due to trion emission, in conjunction with the motion of that magnetization due to spin diffusion. In what follows, we first qualitatively describe these physical processes and explain how they lead to our data, and then we present equations to formally model the dynamics quantitatively.

One important assumption is a separation of dynamics into three very distinct timescales. The fastest timescale is the pulse sequence and resulting electron-spin dynamics, repeated continuously with a repetition period of 143 ns, shown in Fig. 1(b). This is much faster than the nuclear dynamics we consider, which are presumed to occur on millisecond timescales. Finally, the averaging timescale of the measurement is much longer still, on the order of several seconds, allowing the nuclei substantial time to reach quasi-equilibrium.

Processes that change the total nuclear magnetization at the high magnetic fields used here (4 to $10 \mathrm{~T}$ ) are unlikely to be due to the flip-flop terms of the contact hyperfine interaction of the ground-state electron in the QD, as its energy levels are known to be narrow (on the order of $\hbar / T_{2}$, with $T_{2} \sim 3 \mu \mathrm{s}$ ) leaving few viable pathways for energy-conserving nuclear-spin flips. In contrast, the dipolar interaction between a trion's unpaired hole and a nuclear spin may induce a spin-flip with the nuclear Zeeman energy compensated by the broad width of the emitted photon $(\gamma / 2 \pi \sim 0.1 \mathrm{GHz})$. Fermi's golden rule allows an estimate of the rate at which a trion hole (at position $\mathbf{r}_{\mathrm{h}}$, with gyromagnetic ratio $g_{\mathrm{h}}$ ) polarized along the sample growth axis (orthogonal to the magnetic field) randomly flips a nuclear spin at position $\mathbf{r}$ in a spatially flat QD during spontaneous emission, with the photon energy density of states negligibly changed by the Zeeman energy of the nucleus. The result is $\Gamma(\mathbf{r}) \approx$ $\left(9 \mu_{0}^{2} / 128 \pi\right)\left(\mu_{\mathrm{B}} g_{\mathrm{h}} / B_{0}\right)^{2} \gamma\left\langle\left|\mathbf{r}-\mathbf{r}_{\mathrm{h}}\right|^{-3}\right\rangle^{2} \sim 1 /(20 \mathrm{~ms})$, where the brackets refer to an average over the hole wavefunction. Nuclear polarization due to this process has been considered before in the modeling of similar effects $[6,9]$. The change in the nuclear magnetization induced by the trion is random; spins may flip in either direction, leading to a random walk. However, the rate of this walk is itself modified by the probability that the trion is excited. This probability is a function of the pulse sequence; for the sequence shown in Fig. 1(b), we take it as

$$
C(\omega, \tau)=S_{\mathrm{p}}^{z} \frac{[1-\exp (-\beta(\omega) T)]\left[1-\cos \left[\left(\omega_{0}+\omega\right) \tau\right]\right]}{1-\cos \left[\left(\omega_{0}+\omega\right) \tau\right] \exp (-\beta(\omega) T)} .
$$

Here, $\omega_{0}$ is the Larmor frequency of the electron spin in the absence of nuclear shifts, $\omega$ is the randomly drifting Overhauser shift, $\beta(\omega)$ is the rate of optical pumping, $T$ is the pumping time, and $S_{\mathrm{p}}$ is the saturation value of the polarized spin, equal to $1 / 2$ for perfect pumping. 


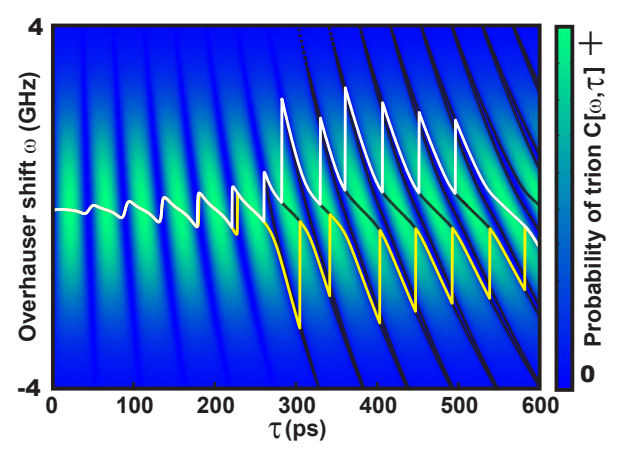

FIG. 2: (Color online) Count-rate $C(\omega, \tau)$ as a function of Overhauser shift $\omega$ and two-pulse delay $\tau$. The lighter grey or greener areas indicate where a higher count-rate is expected. Oscillations in the horizontal directions at frequency $\omega_{0}+\omega$ are due to Ramsey interference; the Gaussian envelope in the vertical direction is due to the reduction of optical pumping with detuning. Superimposed in black is a line indicating where $\partial \omega / \partial t=0$ according to Eq. (4). Superimposed on this line are the solutions to this equation which result as $\tau$ is scanned forward (yellow or light grey) and backward (white).

This function is plotted in Fig. 2. $C(\omega, \tau)$ oscillates sinusoidally with increasing $\tau$ due to the spin's Larmor precession; the Overhauser shift $\omega$ affects the frequency of Larmor precession. For large values of $|\omega|$, the trion transition shifts away from resonance with the optical pumping laser, leading to reduced pumping efficiency and trion creation. If magnetization drift due to trions were the only process, $\omega$ would randomly drift to whatever value is needed to null $C(\omega, \tau)$, thereby slowing and stopping the drift. As a result, no fringes would be observed.

The second process which counters this drift is the presence of nuclear spin diffusion. When trion emission pushes $\omega$ to too large a value, nuclear dipolar interactions "flatten" the nuclear magnetization. As a result, the shift $\omega$ is "pulled" back to a low value, countering the tendency of trion emission to push $\omega$ away from zero. The stable quasi-equilibrium value of $\omega$ resulting from the balance of these processes lives on the edge of the fringes shown in Fig. 22 the nuclear polarization "surfs" along the edge of this function as $\tau$ is changed. As $\tau$ is increased, $|\omega|$ increases causing the observable photon count to decrease due to the reduced degree of optical pumping. When $|\omega|$ is so high that pumping is ineffective $(\beta(\omega) \rightarrow 0)$ and the trion-induced walk stops, spin-diffusion causes the system to drift back to a new stable magnetization at a lower value of $|\omega|$, and the process continues.

These processes may be formally modeled by a diffusion equation for the nuclear distribution. In this model, the nuclear magnetization at each nuclear site $j$ is a random variable, $M_{j}$. A probability distribution function (PDF) $f\left(m_{1}, m_{2}, \ldots ; t\right)=f(\mathbf{m} ; t)$ gives the joint probability that the nuclear magnetization at each position is $M_{j}=m_{j}$ at time $t$. The Overhauser shift is then also a random variable $\Omega$, defined by $\Omega=\sum_{j} A\left(\mathbf{r}_{j}\right) M_{j}$, where
$A\left(\mathbf{r}_{j}\right)$ is the electron hyperfine field at the position $\mathbf{r}_{j}$ of nucleus $j$. The average value of $\Omega$ at time $t$ is written $\langle\Omega\rangle=\omega(t)$ and is found by averaging over all possible values of each $M_{j}$, weighted by the joint PDF $f(\mathbf{m} ; t)$. This joint PDF obeys the equation

$$
\begin{aligned}
\frac{\partial f}{\partial t}=\sum_{j}\left\{-\sum_{k} D_{j k}\right. & \left(\frac{\partial f}{\partial m_{k}}-\frac{\partial f}{\partial m_{j}}\right)+ \\
& {\left.\left[F_{j}+\Gamma\left(\mathbf{r}_{j}\right) C(\Omega, t)\right] \frac{\partial^{2} f}{\partial m_{j}^{2}}\right\} . }
\end{aligned}
$$

The first term, in which the sum over $k$ is the sum over neighbors of $j$, describes the dissipative component of nuclear spin diffusion with diffusion rates $D_{j k}$. The second term describes the random walk of the magnetization at each location $\mathbf{r}$ due to stochastic nuclear spin-flips from the trion hole-spin; the constant $F_{j}$ models the fluctuating component of nuclear spin diffusion, a term needed to understand single QD $T_{2}^{*}$ effects in the absence of the nonlinearities we consider here.

A detail omitted from this model is the degree to which the nuclear spin diffusion is slowed or directed by the presence of the electronic hyperfine field. The magnetic field gradient introduced by the electron does not completely freeze diffusion in this case, because the electronic wave-function is large in comparison to the nuclear-nuclear spacing. Reference 13 indicates methods by which this could be modeled. However, our work solving Eq. (2) including such methods added greater complication and little insight into the observed data.

Remarkably, the data of Fig. 1 can be understood by examining just the average shift $\omega(t)$ using two simplifying assumptions. In the first assumption, the electron wavefunction is modeled as a surface with a hard boundary, and the first term of Eq. (2) acts to decrease $\omega(t)$ by modeling diffusion through that boundary. This results in the equation

$$
\frac{\partial \omega}{\partial t}=-\kappa \omega+\alpha\left\langle\frac{\partial^{2}}{\partial \Omega^{2}}[\Omega C(\Omega, t)]\right\rangle .
$$

The constant $\kappa$ depends on the electronic wavefunction and the rate of nuclear diffusion, but we treat this parameter as adjustable rather than attempting a microscopic description. The constant $\alpha$ is formally given by $\sum_{j} \Gamma\left(\mathbf{r}_{j}\right) A^{2}\left(\mathbf{r}_{j}\right)$.

Unfortunately, Eq. (3) is not a closed system of equations, because it still requires full knowledge of $f(\mathbf{m} ; t)$ to solve. However, if $C(\Omega, \tau)$ is a sufficiently flat function of $\Omega$ in comparison to the width of $f(\mathbf{m} ; t)$, then we may treat $C(\Omega, \tau)$ as roughly constant at $C(\omega(t), \tau)$ over the small width of $f(\mathbf{m} ; t)$. This is our second assumption, leaving the final equation

$$
\frac{\partial \omega}{\partial t}=-\kappa \omega+\alpha \frac{\partial^{2}}{\partial \omega^{2}}[\omega C(\omega, t)] .
$$




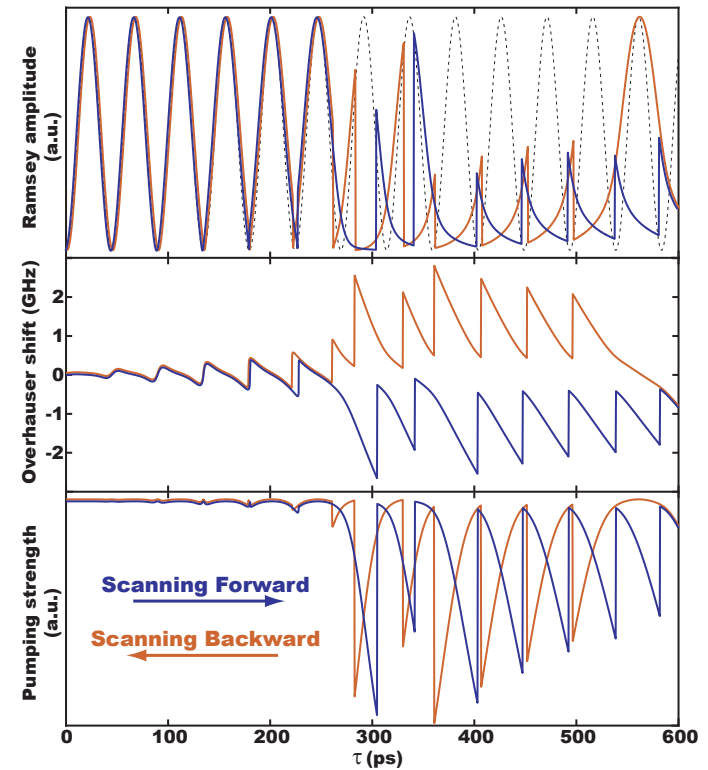

FIG. 3: (Color online) The modeled (a) countrate or Ramsey amplitude $C\left(\omega_{\mathrm{f}}, \tau\right)$, (b) Overhauser shift $\omega_{\mathrm{f}}$, and (c) Pumping strength $\beta\left(\omega_{\mathrm{f}}\right)$. The dotted line in (a) is the expected Ramsey fringe in the absence of nuclear effects. The traces in (b) are the same as those in Fig. 2. The blue or darker grey line corresponds to scanning $\tau$ forward, and the red or lighter grey line corresponds to scanning $\tau$ backward.

Invoking our separation of timescales, we presume $\omega(t)$ evolves from its initial value (set by the last chosen value of $\tau$ ) to a quasi-equilibrium final value $\omega_{\mathrm{f}}$. This final value determines the expected count rate $C\left(\omega_{\mathrm{f}}, \tau\right)$ at this value of $\tau$. We solve by assuming $\omega(0)=0$ at the first attempted value of $\tau$, and then we scan $\tau$ up and then down as in the experiment, finding the steady-state solution of Eq. (4) at each value.

Figure 3 shows the modeled $C\left(\omega_{\mathrm{f}}, \tau\right), \omega_{\mathrm{f}}$, and $\beta\left(\omega_{\mathrm{f}}\right)$ as a function of $\tau$. This particular model used $\kappa / \alpha=10^{4}$, which reproduces the qualitative shape of the data quite well, and quantitatively reproduces the location where sinusoidal fringes evolve into sawtooth-like fringes. Qualitative differences are dominated by the random conditions that develop when the stage is moved on its rail, forming the breaks between data sets in Fig. 11(a). Details of the shape of the waveform are related to the assumed form of the optical absorption. For simplicity, we have used $\beta(\omega)=\beta_{0} \exp \left(-\omega^{2} / 2 \sigma^{2}\right)$, with $\sigma / 2 \pi=1.6 \mathrm{GHz}$ and $\beta_{0}=3 / T$ for known pumping time $T=26 \mathrm{~ns}$, which roughly matches the experimentally observed count-rate when scanning the pump laser across the resonance. The real absorption shape is difficult to observe directly since hysteretic nuclear pumping effects also appear in absorption experiments with scanning $\mathrm{CW}$ lasers, as reported elsewhere [6, 7].

This effect may be useful for future coherent technologies employing QDS. This pulse sequence may serve as a "preparation step" for a qubit to be used in a quantum information processor, as it tunes the qubit to a master oscillator 14 and narrows the random nuclear distribution, assisting more complex coherent control [2, 3, 4, 5, 8, 9, In particular, the ability to control a single electron with effectively $\delta$-function-like rotation pulses introduces strong potential for dynamical decoupling [15, 16, but many schemes, especially those that compensate for pulse errors such as the Carr-Purcell-Meiboom-Gill (CPMG) sequence, require some method to tune the QD's Larmor period to an appropriate division of the pulse-separation time.

In conclusion, we have observed nonlinear nuclear feedback effects in a single charged QD resulting from the countering processes of random nuclear walks driven by trion creation, the finite width of optical absorption, Overhauser-shifted Larmor precession, and nuclear spin diffusion. This feedback may be employed for tuning the electron Larmor period to a particular pulse separation time for more complex pulses sequences. Although the model we have presented is simple and replicates the data well, it is insufficient to describe the processes in a QD under all possible pulse sequences. In particular, these nonlinear effects are highly suppressed in spin-echo measurements [17, even though trion creation follows a similar nonlinear function to Eq. (1). Future work will involve extending this model to explain the non-Markovian effects of nuclei under more complex pulse sequences, as well as exploiting it to extend QD-based quantum memories.

We thank Erwin Hahn for valuable discussions. This work was supported by NICT, MEXT, NSF CCF0829694, and Special Coordination Funds for Promoting Science and Technology. PLM was supported by the David Cheriton Stanford Graduate Fellowship.

* Currently at HRL Laboratories, LLC, 3011 Malibu Canyon Rd., Malibu, CA 90265. Electronic address: tdladd@gmail.com

[1] M. D. Schroer and J. R. Petta, Nat. Phys. 4, 516 (2008).

[2] F. H. L. Koppens et al., Science 309, 1346 (2005).

[3] D. J. Reilly et al., Science 321, 817 (2008).

[4] S. Foletti et al., Nat. Phys. 5, 903 (2009).

[5] I. T. Vink et al., Nat. Phys. 5, 764 (2009).

[6] X. Xu et al., Nature 459, 1105 (2009).

[7] C. Latta et al., Nat. Phys. 5, 758 (2009).

[8] A. Greilich et al., Science 317, 1896 (2007).

[9] S. G. Carter et al., Phys. Rev. Lett. 102, 167403 (2009).

[10] J. Berezovsky et al., Science 320, 349 (2008).

[11] D. Press, T. D. Ladd, B. Y. Zhang, and Y. Yamamoto, Nature 456, 218 (2008).

[12] E. D. Kim et al., arXiv:0910.5189v2.

[13] A. Z. Genack and A. G. Redfield, Phys. Rev. Lett. 31, 1204 (1973).

[14] S. M. Clark, K. M. C. Fu, T. D. Ladd, and Y. Yamamoto, 
Phys. Rev. Lett. 99, 40501 (2007)

[15] G. S. Uhrig, Phys. Rev. Lett. 98, 100504 (2007).

[16] K. Khodjasteh and D. A. Lidar, Phys. Rev. A 78, 012355 (2008).

[17] D. Press, K. D. Greve, P. L. McMahon, T. D. Ladd,
B. Friess, C. Schneider, M. Kamp, S. Höfling, A. Forchel, and Y. Yamamoto, submitted.

[18] Similar data is seen in similar experiments performed elsewhere; see the top trace of Fig. 3b of Ref. 12 